\title{
Phenotypic Characteristics of Yeasts of the Genus Candida and Cryptococcus in Differential Culture Media
}

\author{
Larissa Alves Lima, Mylla Augusta Silva Faria, Ralciane de Paula Menezes, \\ Mário Paulo A. Penatti and Reginaldo dos Santos Pedroso*
}

\author{
Technical Course in Clinical Analysis, Technical School of Health, Federal University of \\ Uberlandia, Campus Umuarama, Uberlandia, Minas Gerais, Brazil
}

*Corresponding author

\begin{tabular}{|l|}
\hline Ke y w o r d s \\
Culture media, \\
Yeasts, \\
Cryptococcus, \\
Candida \\
\hline Article Info \\
\hline $\begin{array}{l}\text { Accepted: } \\
\text { 12 July 2018 } \\
\text { Available Online: } \\
\text { 10 August } 2018\end{array}$ \\
\hline
\end{tabular}

\section{A B S T R A C T}

In the clinical mycology laboratory, the identification of yeast species is done by screening in specific media, such as chromogenic agar for Candida species and Niger seed agar for Cryptococcus species, both of which are of clinical interest. This study aimed to evaluate the growth and morphological characteristics of yeasts of the Candida and Cryptococcus species in different culture media. Yeast species included in the study were: $C$. albicans, C. dubliniensis, C. glabrata, C. tropicalis, C. krusei, C. lipolytica, C. parapsilosis, C. metapsilosis, C. orthopsilosis, C. neoformans, C. gattii, C. flavescens, and C. albidus. The media used were Sabouraud dextrose agar, Sabouraud dextrose broth, hypertonic Sabouraud broth (plus 6.5\% NaCl), Candida chromogenic agar, methyldopa agar, Niger seed agar and tobacco agar. Growth, color, size, presence of fringes, melanin and the appearance of the colony were evaluated. All isolates grew in the media used, except for the hypertonic Sabouraud broth; in Candida chromogenic agar, C. albicans and $C$. dubliniensis present a green color and $C$. tropicalis a blue color, while other species show colors including pink, purple, gray and white; in the Niger seed agar, C. neoformans, $C$. gattii and $C$. flavescens presented a brown color, while others had white colonies; in tobacco agar, the colors included white, cream and gray; and in methyldopa agar, all colonies were white. Some isolates presented colonies with fringes in the tobacco, methyldopa and Niger seed agar; the presence of melanin was observed by Cryptococcus isolates in the Niger seed and tobacco agar; the appearance of colonies in the media varied from opaque to shiny or mucoid, according to the isolate and the culture medium. All of the culture media used allowed the growth of the tested isolates, except for C. lipolytica, which did not grow in hypertonic Sabouraud broth. The isolates of Cryptococcus, C. krusei and $C$. dubliniensis presented a significant reduction of growth in hypertonic Sabouraud broth.

\section{Introduction}

The fungi of the genus Cryptococcus and Candida are among the most isolated in clinical mycology laboratories. They are important fungi for humans and animals due to the diseases that they cause, such as primary or opportunistic mycoses (Kwon- 
Chung and Bennett, 1992; Sidrim et al., 2002).

Cryptococcus species are cosmopolitan and are found in the environment, and associated with bird excreta and different vegetable debris. They are agents of human and animal mycoses, with two main species being involved: Cryptococcus neoformans and Cryptococcus gattii (Kwon-Chung and Bennett, 1992). Some species of Candida, in turn, inhabit the body surface of animals and humans, but have also been isolated from the environment, including the air and water. Candida albicans is the best known species, accounting for the majority of cases of candidiasis (Lacaz et al., 2002).

In the mycology laboratory, the identification of yeasts is performed by screening with classic tests, such as the germ-tube formation test, microculture in corn-tween 80 agar and auxanograms, which are generally more timeconsuming. Other tests that are faster may be included, such as some miniaturized commercial tests that contain colorimetric, biochemical or enzymatic tests; these are more practical, but incur higher costs. The rapid confirmation of a fungal infection is important so that the treatment can be specific and started as soon as possible (Kwon-Chung and Bennett, 1992; Lacaz et al., 2002).

The identification of a fungus from a clinical sample begins with the direct examination of the sample and followed by its isolation in culture. From the culture, the characteristics of the colonies, such as appearance and color, are observed in the specific media for fungal growth, for example Sabouraud agar, chromogenic agar, Niger seed agar and sunflower agar. Subsequently, a microscopic analysis of colonies of these cultures is performed, as well as additional biochemical tests, following the identification algorithm (Kwon-Chung and Bennett, 1992; Lacaz et al., 2002).
Yeasts of the genus Cryptococcus are generally identified by the direct investigation of fungal structure in the clinical material, characterized by the presence of a capsule surrounding the cell, followed by culture. After the isolation of the colonies, the identification is performed as follows: evaluation of the in vitro production of urease, melanin production in media containing phenolic compounds, such as Niger seed agar, and confirmation of the presence of a capsule surrounding the cells. The differentiation between species of this genus is possible through physiological tests such as the assimilation of carbohydrates and nitrogen, from different sources. The species of $C$. neoformans and C. gattii, which are the most frequently isolated, can also be differentiated using bromothymol blue canavanine-glycine agar, in which $C$. neoformans is unable to grow, whereas $C$. gattii grow and change the media color from the original green to an intense blue (Freydiere et al., 2001; Lacaz et al., 2002; Pedroso et al., 2007).

Species of the genus Candida are differentiated in the laboratory by phenotypic tests such as germ tube test, filamentation tests on corn agar plus tween 80, auxanograms and zymograms. The most frequent species in the clinical mycology laboratory are: C. albicans, C. glabrata, $C$. tropicalis, C. parapsilosis, C. krusei and $C$. guilliermondii. Another species, $C$. dubliniensis, although not very frequent, presents phenotypic and biochemical characteristics that are very similar to $C$. albicans, meaning that they need to be differentiated between for epidemiological purposes and also because they can present different responses to antifungals (Kirkpatrick et al., 1998). A more intense green color in the chromogenic agar would be indicative of C. dubliniensis, while other practical tests, such as growth at variable temperatures, and morphology in tobacco agar, among others, 
would be able to differentiate between these species (Freydiere and Guinet, 1997; Loreto et al., 2010; Pasligh et al., 2010). Currently, another species, $C$. auris, has emerged as a resistant species, sometimes presenting resistance to one or more classes of antifungals, which makes its identification and the in vitro susceptibility testing of antifungals extremely important (Arauz et al., 2018; Arendrup and Patterson, 2017).

In the context of the use of culture media for screening and presumptive identification in mycology laboratories, this study aimed to evaluate the growth and morphocolonial characteristics of Candida and Cryptococcus species in different culture media.

\section{Materials and Methods}

\section{Microorganisms and culture media}

Twenty-one isolates of the Candida and Cryptococcus genera, including reference strains (INCQS, ATCC), controls (here called CFP, CP) and environmental (AMB 1) and clinical isolates (the others) used in laboratory, were evaluated. Specifically, the isolates studied were: three isolates of Candida parapsilosis (INCQS 40038, ATCC 22019 and CP 110), one of Candida metapsilosis (CP 111), one of Candida orthopsilosis (CP 112), two of Candida albicans (ATCC 90028 and P02), three of Candida dubliniensis (C142, P55V and P63), one of Candida tropicalis (P29R), two of Candida krusei (P61 and P115Rx), two of Candida glabrata (SV27 and SV113), one of Candida lipolytica (SV123), one isolate of Cryptococcus flavescens (AMB 1), one of Cryptococcus albidus var. albidus (INCQS 40190), one of Cryptococcus gattii (CFP 62), one of Cryptococcus neoformans var. neoformans (ATCC 28957) and one of Cryptococcus neoformans var. grubii (ATCC 90112).
Prior to the execution of the tests, samples stored in BHI-glycerol, kept at $-20^{\circ} \mathrm{C}$, were spiked onto Sabouraud agar and incubated at $30^{\circ} \mathrm{C}$ for $48-72 \mathrm{~h}$; afterwards, two more seedings were performed on the same media for the complete reactivation of isolates and then the tests were performed.

The culture media tested were: Sabouraud dextrose agar (SDA), Niger seed agar (NSA), tobacco agar (TBCA), methyldopa agar (MDA), chromogenic agar for Candida (CHRmA), Sabouraud dextrose broth (SDB) and hypertonic Sabouraud dextrose broth (HSDB, which is the SDB plus 6.5\% NaCl).

\section{In vitro tests}

Suspensions of each isolate were made in sterile saline $(\mathrm{NaCl} 0.9 \%)$, with turbidity equivalent to 0.5 tube of the McFarland scale, prepared from a 48-hour culture on ASD.

From those suspensions, 10 microliters of each were seeded on the surface of agar contained in petri dishes $(90 \times 15 \mathrm{~mm})$. This was performed for each of the culture media (NSA, TBCA, MDA and CHRmA). Seeding was undertaken on four plates of each culture medium. Subsequently, the plates were incubated at $30^{\circ} \mathrm{C}$ for four days, with daily growth monitoring. The characteristics analyzed in all of the plaques were: growth, color and size of the colonies, formation of fringes in the periphery of the colonies and the production of melanin in the Niger seed, tobacco and methyldopa agar.

Growth tests on SDB and HSDB were performed by transferring $20 \mu \mathrm{l}$ of the suspension of each isolate in saline solution to two tubes containing SBD or HSDB (in duplicate). The tubes were incubated at $30^{\circ} \mathrm{C}$ for four days. SDB was used as a growth and turbidity control for comparison with HSDB. After incubation, the cultures were visually 
examined for turbidity of the broth, which was compared to the McFarland scale tubes, and the test results were expressed according to turbidity.

\section{Results and Discussion}

All isolates of the genera Candida and Cryptococcus were grown on the media agar, methyldopa, tobacco and chromogenic Candida agar (Table 1).

In MDA, all colonies were white, except for C. neoformans and C. gattii, which were light brown. Regarding the size of the colonies, all colonies were small, except for $C$. albidus and $C$. neoformans, which had larger colonies when compared to those formed in SDA.

In the NSA, most of the isolates presented smaller colonies than in the SDA, with the exception of five isolates: $C$. krusei, $C$. flavescens, C. albidus and both $C$. neoformans isolates, whose colonies were similar in size to those observed in SDA. Fringe formation was observed in four isolates: $C$. flavescens, $C$. gattii and the two $C$. neoformans. Melanin production was observed in the colonies of $C$. flavescens, $C$. gattii and the two $C$. neoformans isolates.

In TBCA, all isolates had smaller colonies than those in SDA, ranging from bright or opaque to mucoid, and white, gray or beige to brown colors. Melanin was evidenced in two isolates of $C$. neoformans and one $C$. gattii. One isolate of $C$. dubliniensis and one of $C$. krusei were able to form fringes in TBCA.

In CHRmA, the colors of the colonies ranged from purple, green, beige, blue, white, and pink to gray; most of the isolates presented small colonies in comparison to the SDA and no isolates formed fringes. In addition, the colonies presented variable aspects, and all colonies of $C$. glabrata and Cryptococcus spp. presented a brilliant appearance. In SDB, all isolates of Candida and Cryptococcus presented growth. In HSDB, the isolate of $C$. lipolytica did not show growth, while the Cryptococcus flavescens isolate also presented film formation on the broth surface (Table 2).

The detailed characteristics of the isolates tested are shown in Tables 1 and 2.

In this study, morphological characteristics of Candida and Cryptococcus species were evaluated in different culture media. These yeasts are clinically important because they are capable of causing serious diseases in humans, especially in immunocompromised individuals (Khawcharoenporn et al., 2007; Pfeiller and Diekema, 2004).

The ideal culture medium for fungal growth and development is one that has all of the necessary substrates for the in vitro reproduction of microorganisms such as nitrogen, carbon, micronutrients, water, and others. For fungi, unlike bacteria, the isolation and identification of the main species can only be carried out with a few culture media in microbial laboratories (Lacaz et al., 2002).

Some culture media are widely used, with different purposes such as: screening, selecting and differentiating specific isolates and the preliminary identification of isolates present in clinical samples; these include Sabouraud dextrose agar, corn agar, chromogenic agar, Niger seed agar, tobacco agar, methyldopa agar and hypertonic Sabouraud dextrose broth (Freydiere and Guinet, 1997; Loreto et al., 2010; Menezes et al., 2011; Pasligh et al., 2010). Niger seed agar, for instance, is a selective and differential medium for the species Cryptococcus neoformans and C. gattii, which show colonies with brown pigment (Kwon-Chung and Bennett, 1992). 


\begin{tabular}{|c|c|c|c|c|c|c|c|c|c|c|c|c|}
\hline \multirow[b]{2}{*}{ Species / isolate } & \multicolumn{3}{|c|}{ Niger seed agar } & \multicolumn{3}{|c|}{ Methyldopa agar } & \multicolumn{3}{|c|}{ Tobacco agar } & \multicolumn{3}{|c|}{ Chromogenic agar } \\
\hline & Color & Size & Aspect & Color & Size & Aspect & Color & Size & Aspect & Color & Size & Aspect \\
\hline Candida parapsilosis INCQS 40038 & White & Tiny & Bright & White & Tiny & Bright & White & Tiny & Bright & Pink & Small & Bright \\
\hline C. parapsilosis ATCC 22019 & White & Tiny & Bright & White & Tiny & Bright & Beige & Small & Bright & Purple & Small & Opaque \\
\hline C. parapsilosis CP 110 & White & Tiny & Bright & White & Tiny & Bright & White & Tiny & Bright & Purple & Large & Opaque \\
\hline C. metapsilosis CP 111 & White & Tiny & Bright & White & Tiny & Opaque & White & Tiny & Opaque & Purple & Small & Bright \\
\hline C. orthopsilosis CP 112 & White & Small & Bright & White & Tiny & Bright & White & Tiny & Bright & Beige & Small & Bright \\
\hline C. albicans ATCC 90028 & White & Tiny & Opaque & White & Small & Bright & White & Tiny & Opaque & Light green & Small & Bright \\
\hline C. albicans $\mathrm{P02}$ & White & Small & Bright & White & Small & Opaque & Beige & Tiny & Opaque & Light green & Small & Opaque \\
\hline C. dubliniensis $\mathrm{C142}$ & White & Small & Bright & White & Small & Opaque & Beige & Tiny & Opaque & Green & Small & Opaque \\
\hline C. dubliniensis P55V & White & Tiny & Opaque & White & Tiny & Opaque & Beige & Small & Bright & Green & Small & Bright \\
\hline C. dubliniensis P63 & White & Small & Opaque & White & Tiny & Opaque & Beige & Small & Opaque & Green & Small & Bright \\
\hline C. tropicalis P29R & White & Small & Bright & White & Tiny & Opaque & White & Tiny & Bright & Blue & Large & Opaque \\
\hline C. krusei P61 & White & Small & Opaque & White & Tiny & Opaque & Grey & Tiny & Opaque & Beige & Large & Opaque \\
\hline C. krusei P115Rx & White & Large & Bright & White & Small & Opaque & Beige & Tiny & Opaque & Purple & Large & Opaque \\
\hline C. glabrata SV27 & White & Small & Bright & White & Tiny & Bright & White & Tiny & Opaque & White & Small & Bright \\
\hline C. glabrata SV113 & White & Tiny & Bright & White & Small & Opaque & White & Tiny & Opaque & Purple & Small & Bright \\
\hline C. lipolytica SV123 & White & Small & Mucoid & White & Small & Opaque & White & Small & Opaque & Purple & Small & Opaque \\
\hline Cryptococcus flavescens AMB 1 & Light brown & Large & Mucoid & White & Small & Bright & Beige & Small & Bright & Pink & Small & Bright \\
\hline C. albidus INCQS 40190 & White & Large & Bright & White & Large & Bright & Beige & Small & Bright & Pink & Small & Bright \\
\hline C. gattii CFP 62 & Brown & Small & Mucoid & White & Small & Bright & Light brown & Tiny & Opaque & Pink & Small & Bright \\
\hline C. neoformans ATCC 28957 & Brown & Large & Mucoid & White & Small & Bright & Light brown & Tiny & Opaque & Grey & Small & Bright \\
\hline C. neoformans ATCC 90112 & Light brown & Large & Mucoid & White & Large & Bright & Brown & Small & Mucoid & Pink & Small & Bright \\
\hline
\end{tabular}


Table.2 Characteristics of the growth of Candida spp. and Cryptococcus spp. in hypertonic Sabouraud broth

\begin{tabular}{|c|c|c|c|c|}
\hline Species & $\begin{array}{l}\text { Growth in } \\
\text { SDB* }\end{array}$ & $\begin{array}{l}\text { Growth in } \\
\text { HSDB* }\end{array}$ & $\begin{array}{l}\text { Growth on } \\
\text { SDB surface }\end{array}$ & $\begin{array}{l}\text { Growth on } \\
\text { HSDB surface }\end{array}$ \\
\hline Candida parapsilosis INCQS 40038 & 3 & 3 & 0 & 0 \\
\hline Candida parapsilosis ATCC 22019 & 5 & 4.5 & 0 & 0 \\
\hline Candida parapsilosis CP 110 & 4 & 3 & 0 & 0 \\
\hline Candida metapsilosis CP 111 & 6 & 5 & 0 & 0 \\
\hline Candida orthopsilosis CP 112 & 5 & 5 & 0 & 0 \\
\hline Candida albicans ATCC 90028 & 5 & 4 & 0 & 0 \\
\hline Candida albicans $\mathbf{P 0 2}$ & 5 & 4 & 0 & 0 \\
\hline Candida dubliniensis C142 & 7 & 1 & 0 & 0 \\
\hline Candida dubliniensis P55V & 7 & 0.5 & 0 & 0 \\
\hline Candida dubliniensis P63 & 7 & 0.5 & 0 & 0 \\
\hline Candida tropicalis P29R & 6 & 5 & 0 & 0 \\
\hline Candida krusei P61 & 5 & 0.5 & 1 & 0 \\
\hline Candida krusei P115Rx & 7 & 0.5 & 1 & 0 \\
\hline Candida glabrata SV27 & 8 & 6 & 0 & 0 \\
\hline Candida glabrata SV113 & 6.5 & 5.5 & 0 & 0 \\
\hline Candida lipolytica SV123 & 7 & 0 & 1 & 0 \\
\hline Cryptococcus flavescens AMB 1 & 3 & 0.5 & 1 & 1 \\
\hline Cryptococcus albidus var. albidus INCQS 40190 & 4 & 0.5 & 0 & 0 \\
\hline Cryptococcus gattii CFP 62 & 3 & 0.5 & 0 & 0 \\
\hline $\begin{array}{l}\text { Cryptococcus neoformans var. neoformans ATCC } \\
28957\end{array}$ & 1 & 0.5 & 0 & 0 \\
\hline Cryptococcus neoformans var. grubii ATCC 90112 & 4 & 0.5 & 0 & 0 \\
\hline
\end{tabular}

Note: *: according to turbidity scale of McFarland; 0: Absent / negative test; SDB: Sabouraud Dextrose Broth; HSDB: hypertonic SDB 
Tobacco agar is another medium used to verify the production of the melanin pigment in Cryptococcus species, but also for the differentiation between Candida albicans and C. dubliniensis (Loreto et al., 2010; SilveiraGomes et al., 2011).

Chromogenic agar is a selective and differential medium for yeasts of the genus Candida, allowing differentiation between some species according to the coloration of the colonies; for example, the colonies of $C$. albicans and $C$. dubliniensis present a green color, while colonies of $C$. tropicalis show a blue color, colonies of $C$. krusei are lilac and dry, whereas other species may have white, cream or gray coloration (Odds and Bernaerts, 1994; Rousselle et al., 1994).

In the present study, all isolates of Cryptococcus spp. grew in the NSA and TBCA. Regarding melanin expression, only the Cryptoccocus albidus var. albidus was unable to produce this on TBCA; however, a brownish pigment was evidenced in NSA. The production of melanin in specific media by other species of Cryptococcus besides $C$. neoformans and $C$. gattii has been demonstrated in the literature (Menezes et al., 2011; Pedroso et al., 2007). None of the Candida species presented melanin in these media. The interesting thing in these findings was the production of melanin by $C$. flavescens in the NSA, a fact that has not yet been described in the literature, and which could be elucidated when the metabolic pathways related to the expression of laccase and phenol oxidase-like enzymes are studied and described.

MDA was proposed as a minimum, chemically controlled medium for the expression of melanin in Cryptococcus spp. (Menezes et al., 2011). The melaninproducing species presented a very light brown color, which it is difficult to verify, and is only possible when compared with an isolated control that does not express the pigment. On this culture medium it is interesting to note that $\mathrm{pH}$ seems to play a key role in the expression of melanin, since the pigment is evidenced at $\mathrm{pH}$ between 5.0-5.5. This medium only provided evidence of fringes for the species $C$. tropicalis and $C$. krusei, with no evidence of other characteristics that would be useful for the screening of other species in a routine laboratory.

The formation of fringes around colonies was observed in a few isolates in the media TBCA, MDA and NSA. This characteristic is presented by some isolates, but is dependent on the species and medium; therefore, it may not be a characteristic that can be used in the screening of microorganisms in the laboratory. However, it is a feature that can be used in studies which include morphotyping (Bacelo et al., 2010).

TBCA was proposed as a form of differentiation between $C$. albicans and $C$. dubliniensis. According to the literature, colonies of $C$. dubliniensis in TBCA, as well as in NSA, have fringes due to the abundant mycelium and numerous chlamydospores; this does not occur with colonies of $C$. albicans, which are smooth and do not produce chlamydospores in those media (Liverio et al., 2017; Loreto et al., 2010). Contrary to the expected results, in the present study, however, only colonies of one of the three isolates of $C$. dubliniensis showed fringes, which suggests that this characteristic needs to be further explored and studied to determine the optimal incubation conditions and the interfering factors.

The development of Candida chromogenic agar was an evolution in relation to culture media, as it enabled the laboratories to identify $C$. albicans, the most frequent species 
in clinical samples, with a high degree of certainty (Odds and Bernaerts, 1994). In general, in this study, the isolates showed different morphological aspects, ranging from bright to opaque. The results of this analysis are in agreement with the expected results and those which have been widely described in the literature. The differentiation between $C$. albicans and $C$. dubliniensis in chromogenic agar has been suggested by some authors (Liverio et al., 2017); however, it seems to be difficult, and only when isolates from these two species are cultivated simultaneously is it possible to perceive the variation in the shade of green produced, according to the author's experience. On the other hand, different chromogenic culture media of different brands are on the market, and their performance is different, as shown by recent studies (Vecchione et al., 2017). Therefore, it is up to each laboratory to evaluate the benefits and costs, and to adopt the method that best meets their needs.

The hypertonic Sabouraud dextrose broth was proposed to differentiate $C$. albicans from $C$. dubliniensis, as the latter shows inhibition or an expressive reduction of growth (Mahelová and Ruzicka, 2017; Silveira-Gomes et al., 2011). In the present study, it was observed that many isolates had a decrease in growth in HSDB, compared to SDB, according to the turbidity scale. However, according to the literature, $C$. dubliniensis presented a decrease when compared to the McFarland turbidity scale; the three isolates of $C$. dubliniensis presented turbidity equivalent to tube 7 in the SDB, while the turbidity in HSDB ranged from 0.5 to 1 . In HSDB, isolates of C. krusei and $C$. lipolytica showed no growth or presented significant growth reduction. Cryptococcus isolates showed variable growth in SDB, and decreased growth in HSDB. As the isolates of Cryptococcus are aerobic, it is expected that there is little growth in static incubation in liquid medium, but it was possible to verify a reduction of growth in the hypertonic broth. Cryptococcus species require the presence of oxygen for growth, so they will have difficulty growing in liquid media (Kwon-Chung and Bennett, 1992). This is because the culture medium is a suspension of microorganisms; when inoculated, the cells tend to go to the bottom of the tube, so the absence of oxygen will reduce or impede growth. Incubation of the culture media in this study was performed in a static manner; however, if the incubator is shaken, this interference can be eliminated.

Also, it was observed that the isolates of Candida krusei, Candida lipolytica and Cryptococcus flavescens showed a growth film on the surface of the broth in SDB. This has not been previously described, and may help the laboratory in identify those species. In general, all of the media allowed the growth of the tested isolates, with the exception of $C$. lipolytica, which did not grow in HSDB. On the other hand, the isolates of Cryptococcus, C. krusei and C. dubliniensis showed a significant reduction of growth in HSDB.

Phenotypic methods remain the main way to identify or screen fungi that are isolated in the medical laboratory, especially in small laboratories, with a small sample processing capacity, and which are located far from major centers. More advanced methodologies such as those based on DNA or proteomics are restricted to large laboratories and/or reference centers because of the high cost and complexity of the techniques. Thus, studies that seek to improve the screening of species in culture media already used in the routine laboratory, as well as the testing of other media should be encouraged and disseminated, so that the most frequent fungal species in mycology laboratories can be identified with greater accuracy. 


\section{Acknowledgments}

The authors are grateful to the Pro-rectory of Graduation of the Federal University of Uberlandia for the Professional Education Scholarships to L.A. Lima and M.A.S. Faria (Edital number 001/2017/PROGRAD/ DIREN); to Dr Regina Helena Pires, from Franca University, Franca city, São Paulo, Brazil, for providing the isolates of $C$. parapsilosis sensu stricto, $C$ metapsilosis and $C$. orthopsilosis; to Dr Luciana Trilles from Mycology Laboratory, National Institute of Infectious Diseases Evandro Chagas, Oswaldo Cruz Foundation, Rio de Janeiro city, Rio de Janeiro, Brazil, for providing the isolate of $C$. gattii CFP 62; and to National Institute for Health Quality Control (INCQS), Oswaldo Cruz Foundation, Rio de Janeiro city, Rio de Janeiro, Brazil for providing the isolates of $C$ albicans ATCC 90028, C. albidus INCQS 40190 and $C$. parapsilosis INCQS 40038.

\section{References}

Araúz, A.B., Caceres, D.H., Santiago, E., Armstrong, P., Arosemena, S., Ramos, C., et al., 2018. Isolation of Candida auris from 9 patients in Central America: importance of accurate diagnosis and susceptibility testing. Mycoses. 61(1), 44-47.

Arendrup, M.C., and Patterson, T.F. 2017. Multidrug-resistant Candida: epidemiology, molecular mechanisms, and treatment. J. Infect. Dis. 216 (uppl. 3): S445-S451.

Bacelo, K.L., Da Costa, K.R.C., Ferreira, J.C., and Candido, R.C. 2010. Biotype stability of Candida albicans isolates after culture storage determined by randomly amplified polymorphic DNA and methods. Mycoses, 53(6), 468-474.

Ellepola, A.N.B., Hurst, S.F., Elie, C.M., and Morrison, C.J. 2003. Rapid and unequivocal differentiation of Candida dubliniensis from other Candida species using species-specific DNA probes: comparison with phenotypic identification methods. Oral Microbiol. Immunol. 18(6), 379-388.

Faria, G.O., Martins, A.G., Amante-Penatti, M.P., and Pedroso, R.S. 2014. In vitro characteristics of isolates of Cryptococcus laurentii complex in different culture media. Rev. Pat. Trop. 43 (3), 290-302. [In Portuguese].

Freydiere, A.M., and Guinet, R. 1997. Rapid methods for identification of the most frequent clinical yeast. Rev. Iberoam. Micol. 14 (3), 85-89.

Freydiere, A.M., Guinet, R., and Boiron, P. 2011. Yeast identification in the clinical microbiology laboratory: phenotypic methods. Sabouraudia. 39(1), 9-33.

Khawcharoenporn, T., Apisarnthanarak, A., and Mundy, L.M. 2007. Non-neoformans cryptococcal infections: a systematic review. Infection. 35(2), 51-58.

Kirkpatrick, W.R., Revankar, S.G., Mcatee, R.K., Lopez-Ribot, J.L., Fothergill, A.W., McCarthy, D.I., et al., 1998. Detection of Candida dubliniensis in oropharyngeal samples from human immunodeficiency virus-infected patients in North America by primary CHROMagar Candida screening and susceptibility testing of isolates. J. Clin. Microbiol. 36(10), 3007-3012.

Koehler, A.P., Chu, K.C., Houang, E. T., and Cheng, A. F. 1999. Simple, reliable, and cost-effective yeast identification scheme for the clinical laboratory. J. Clin. Microbiol. 37(2), 422-426.

Kwon-Chung, K.J., and Bennett, J.E. 1992. Medical mycology. Lea \& Febiger, Philadelphia, 867p.

Lacaz, C.S., Porto, E., Martins, J.E.C., HeinsVaccari, E.M., and Melo, T. 2002. Tratado de Micologia Médica, Ninth ed. Sarvier, São Paulo, 1104 p. [In Portuguese]

Livério, H.O., Ruiz, L.D.S., Freitas, R.S.D., Nishikaku, A., Souza, A.C.D., Paula, C.R., et al., 2017. Phenotypic and genotypic detection of Candida albicans and Candida dubliniensis strains 
isolated from oral mucosa of AIDS pediatric patients. Rev. Inst. Med. Trop. Sao Paulo. 59, e14.

Loretto, E.S., Scheid, L.A., Nogueira, C.W., Zeni, G., Santurio, J.M., and Alves, S.H. 2010. Candida dubliniensis: epidemiology and phenotypic methods for identification. Mycopathologia. 169(6), 431-443.

Mahelová, M. and Růžička, F. 2017. Methods of Candida dubliniensis identification and its occurrence in human clinical material. Folia Microbiologica, 62(5), 401-408.

Menezes, R.P., Penatti, M.P.A., and Pedroso, RS. 2011. Different culture media containing methyldopa for melanin production by Cryptococcus species. Rev. Soc. Bras. Med. Trop. 44(5), 591594.

Odds, F.C and Bernaerts, R.I.A. 1994. CHROMagar Candida, a new differential isolation medium for presumptive identification of clinically important Candida species. J. Clin. Microbiol. 32(8), 1923-1929.

Pasligh, J., Radecke, C., Fleischhacker, M., and Ruhnke, M. 2010. Comparison of phenotypic methods for the identification of Candida dubliniensis. J. Microbiol. Immunol. Infect. 43(2), 147-154.

Pedroso, R.S., Costa, K.R.C., Ferreira, J.C., and Candido, R.C. 2007. Evaluation of melanin production by Cryptococcus species in four different culture media. Rev. Soc. Bras. Med. Trop. 40(5), 566568.
Pedroso, R.S., Ferreira, J.C., and Candido, R.C. 2009. The isolation and characterization of virulence factors of Cryptococcus spp. from saprophytic sources in the city of Ribeirão Preto, São Paulo, Brazil. Microbiol. Res. 164(2), 221-227.

Pfaller, M.A., and Diekema, D.J. 2004. Rare and emerging opportunistic fungal pathogens: concern for resistance beyond Candida albicans and Aspergillus fumigatus. J. Clin. Microbiol. 42(10), 4419-4431.

Rousselle, P., Freydiere, A. M., Couillerot, P. J., De Montclos, H., and Gille, Y. 1994. Rapid identification of Candida albicans by using Albicans ID and fluoroplate agar plates. J. Clin. Microbiol. 32(12), 3034-3036.

Sidrim, J.J.C. and Moreira, J.L.B. Fundamentos clínicos e laboratoriais da micologia médica. Guanabara Koogan, Rio de Janeiro. 1999. 287p. [In Portuguese].

Silveira-Gomes, F., Sarmento, D.N., EspíritoSanto, E.P.T., Souza, N.O., Pinto, T.M., and Marques-da-Silva, S.H. 2011. Differentiation between Candida albicans and Candida dubliniensis using hypertonic Sabouraud broth and tobacco Agar. Rev. Soc. Bras. Med. Trop. 44(4), 457-460.

Vecchione, A., Florio, W., Celandroni, F., Barnini, S., Lupetti, A., and Ghelardi, E. 2017. Comparative evaluation of six chromogenic media for presumptive yeast identification. J. Clin. Pathol. 70(12), 1074-1078.

\section{How to cite this article:}

Larissa Alves Lima, Mylla Augusta Silva Faria, Ralciane de Paula Menezes, Mário Paulo A. Penatti and Reginaldo dos Santos Pedroso. 2018. Phenotypic Characteristics of Yeasts of the Genus Candida and Cryptococcus in Differential Culture Media. Int.J.Curr.Microbiol.App.Sci. 7(08): 1912-1921. doi: https://doi.org/10.20546/ijcmas.2018.708.220 